\title{
A PATHWAY FOR NUTRIENTS FROM THE MEDULLARY CAVITY TO THE ARTICULAR CARTILAGE OF THE HUMAN FEMORAL HEAD
}

\author{
A. S. Greenwald and D. W. Haynes, Oxford, England \\ From the Department of Engineering Science, Oxford University, and the \\ Nuffield Department of Orthopaedic Surgery, Nuffield Orthopaedic Centre, Oxford
}

It has long been known that articular cartilage does not contain blood vessels, and the mechanism by which nutrients reach the cartilage has been a problem of continued interest. There are two schools of thought as to the pathways by which adult articular cartilage receives its nutrition. One states that nutrients are supplied to the cartilage solely through contact with synovial fluid. This pathway was suggested by early investigators and more recently confirmed (Brower, Akahoshi and Orlic 1962; Maroudas, Bullough, Swanson and Freeman 1968; Hodge and McKibbin 1969). The other school contends that nutrients are supplied not only from the synovial fluid but also from the medullary cavity of the bone supporting the articular cartilage. This pathway has been inferred from studies in rabbits (Holmdahl and Ingelmark 1950, Ekholm 1951, Brodin 1955) and more recently in lambs (McKibbin and Holdsworth 1966). There are no observations on record which offer visual support that this pathway actually exists in adult human joints.

Pathological states in human joints commonly lead to the deposition of fibrous tissue on the surface of the articular cartilage. Rheumatoid arthritis (Fig. 1) and chronic haemarthroses

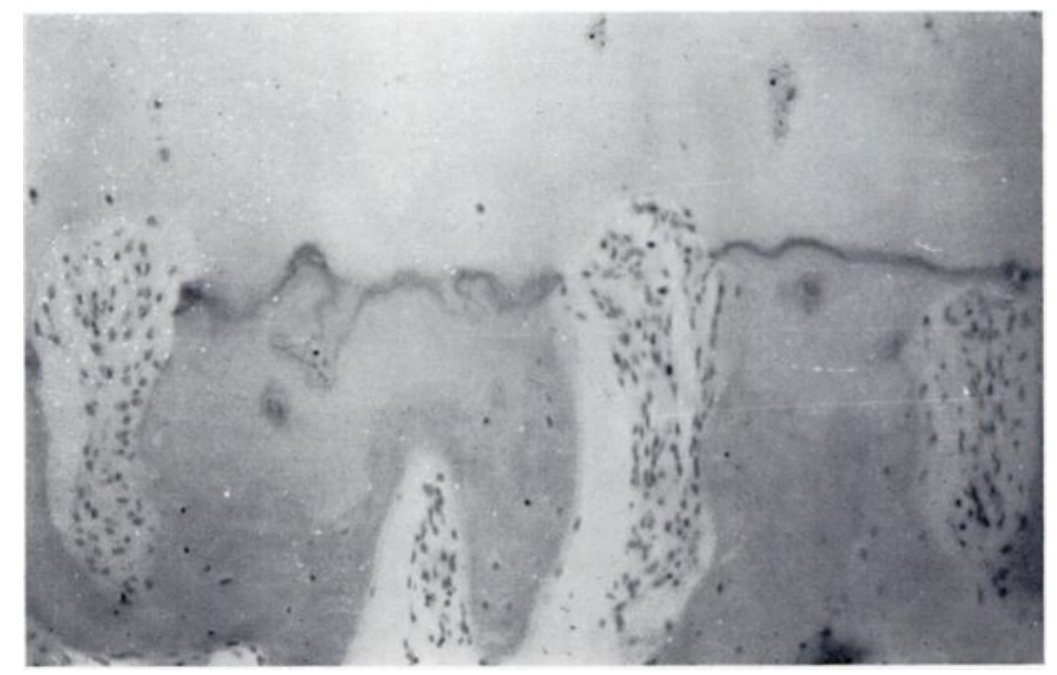

FIG. 1

A decalcified paraffin section from a case of human rheumatoid arthritis. Vascular connective tissue extends from the medullary cavity through the subchondral bone. calcified cartilage and "calcified line" into the articular cartilage. (Haematoxylin and eosin, $\times 120$.)

are examples. Histological examination of joint surfaces on which pannus has formed usually shows that the articular cartilage remains viable although eroded beneath the fibrous tissue. It is also usual to find numerous defects in the subchondral plate and calcified cartilage, forming channels which are filled by vascularised medullary soft tissue and are in contact with the articular cartilage. In a study of the normal adult human hip joint it was noted that similar vascular channels extend through the subchondral plate and make direct contact 
with the cartilage at many points over the whole area of the femoral articulation (Fig. 2) (Woods, Greenwald and Haynes, in press). These observations led us to investigate the possibility that a route of nutrition exists in apparently normal joints from the medullary cavity to the articular cartilage. The femoral head was selected as the structure to study by a dye migration technique using a non-toxic fluorescent dye.

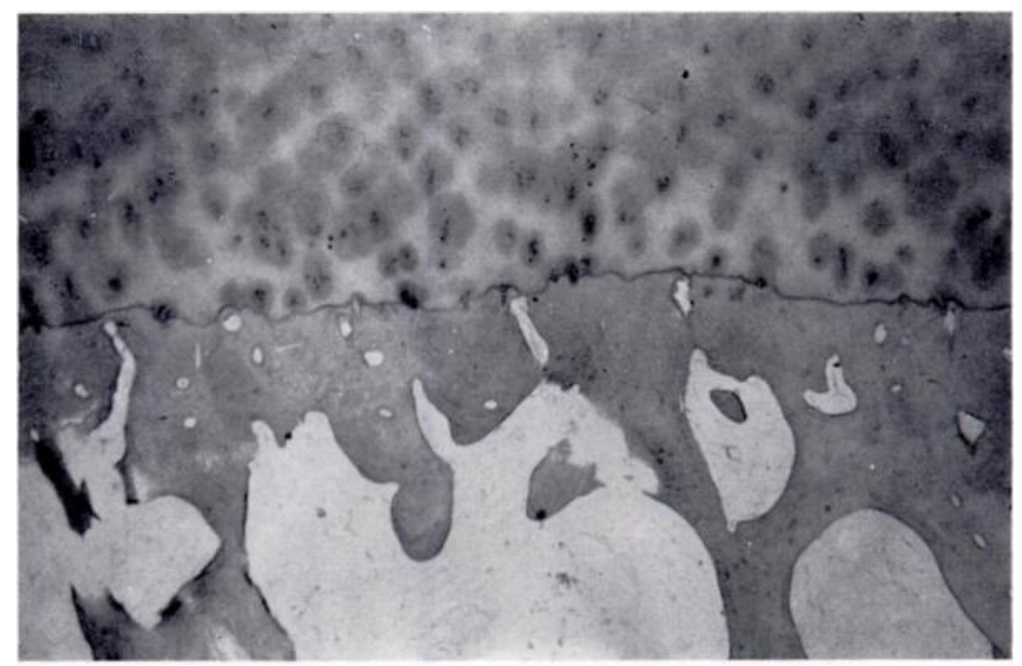

Fig. 2

A decalcified paraffin section from a normal human adult femoral head. Vascular channels extend from the medullary cavity to the "calcified line" at the base of the articular cartilage. (Haematoxylin and eosin, $\times 30$.)

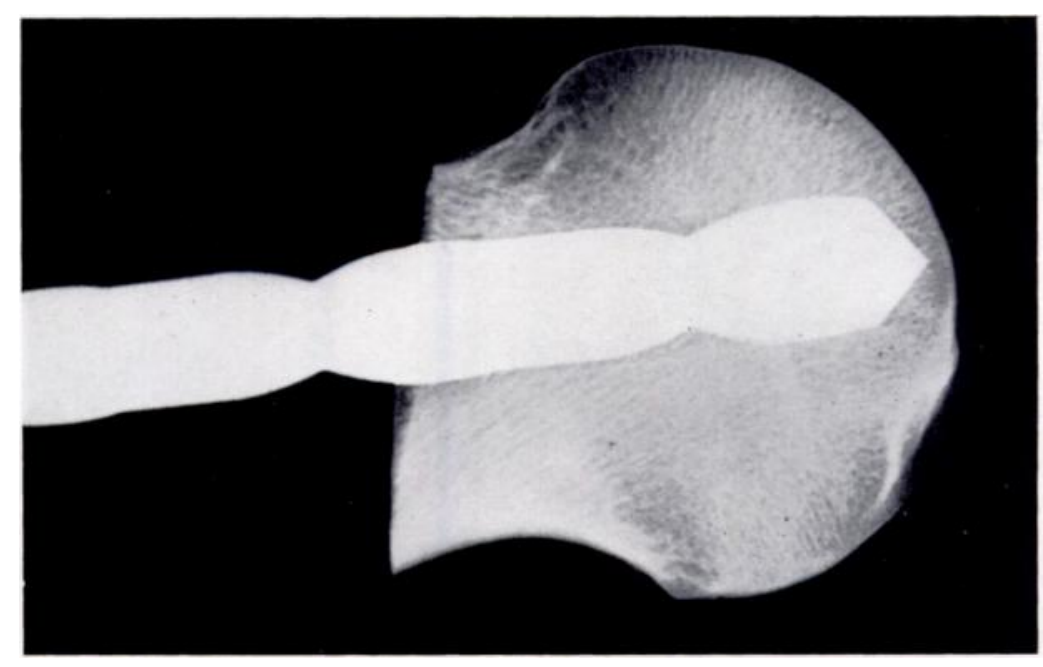

Fig. 3

A radiograph of a human femoral head and drill. The depth of the hole was controlled by a series of radiographs.

\section{MATERIALS AND METHODS}

Twenty fresh human hip joints obtained at necropsy were disarticulated and the femoral head was severed from the shaft approximately at right angles across its neck. A hole was bored into each femoral head with an 11 millimetre steel drill. The hole extended to within three to five millimetres of the subchondral bone and its depth was controlled by a series of 
radiographs (Fig. 3). The drilled head was supported, with the hole vertical, in a container by using cotton wool saturated with normal saline. A 5 per cent solution of fluorescent dye, sodium-3 oxyprene-5, 8, 10 tri-sulphonate (Bayer), in normal saline was introduced into the drill hole. The drilled femoral heads were then stored at 4 degrees Centigrade for sixteen, twenty-four and forty-eight hour periods.

The femoral head was frozen to -70 degrees Centigrade and sawed along the drill hole. The area of cartilage and subchondral bone beneath the drill hole, while still frozen, was cut into slices approximately 5 millimetres thick and attached to metal chucks with ice. These were inserted into a cryostat containing a Cambridge rocking microtome and $15 \mu$ sections were cut. The sections were attached to fluoro-free slides and cover slips secured with D.P.X. mountant. The sections were examined on a Zeiss photomicroscope using ultra-violet light with a BG 3/4 exciter filter and 50/44 barrier filter. Tungsten polarised light was also used to identify the precise location of the dye.

Three further normal human acetabula were drilled in two areas. The dye was allowed to penetrate for sixteen hours and sections prepared and examined under the same conditions as described above.

One femoral head in which osteoarthritic changes were present was also examined by the same technique, allowing forty-eight hours for dye penetration.

Four millilitres of sulphur ${ }^{35}$ in an aqueous solution of 5 millicuries per millilitre was used as a tracer in a group of drilled femoral heads in an attempt to verify the route of the fluorescent dye. Each head was given a forty-eight-hour penetration period, frozen sections prepared as previously described. Autoradiographs were prepared from the sections using Kodak AR-10 stripping film and exposed for periods of up to seven days.

An in vivo experiment was performed to test whether a perfusion technique would significantly alter the results obtained. Under ether anaesthesia the femoral heads of a group of eight-month-old rabbits were dislocated by dividing the capsule and synovium at the brim of the acetabulum and cutting the ligamentum teres. The leg was rotated and the head isolated from the surrounding tissue and kept moist with a continuous saline drip. The opposite femoral head was left intact to serve as a control. The animals were injected intravenously with 20 millilitres of 20 per cent fluorescent dye. Anaesthesia was maintained for periods up to two hours after which the animals were killed. The experimental and control femoral heads were prepared and viewed as previously described.

\section{RESULTS}

In the human and rabbit materials using the fluorescent dye a yellow/green fluorescence was observed in the articular cartilage, osteoid, osteocyte lacunae and canaliculi. The bone matrix and calcified cartilage displayed no affinity for the dye.

In the human femoral head the fluorescent dye after forty-eight hours had passed from the medullary cavity into the articular cartilage and had reached its joint surface (Fig. 4). After sixteen and twenty-four hours the dye had penetrated only a short distance into the articular cartilage adjacent to the calcified cartilage. Greatest intensity and deepest penetration of the dye was observed in areas where vascular spaces were near or in contact with the articular cartilage (Figs. 5 to 7 ).

Sections of the osteoarthritic femoral head revealed a significant decrease in the number of vascular channels present. Dye migration after forty-eight hours' penetration was slight and had only occurred in areas where there were vascular channels (Fig. 8).

In the group of human acetabula the subchondral bone plates were considerably thicker and had fewer vascular channels than the femoral heads. The dye had penetrated only a short distance into the articular cartilage and only in areas corresponding to the vascular channels (Fig. 9).

VOL. 51 B, NO. 4, NOVEMBER 1969

$\mathrm{L}$ 


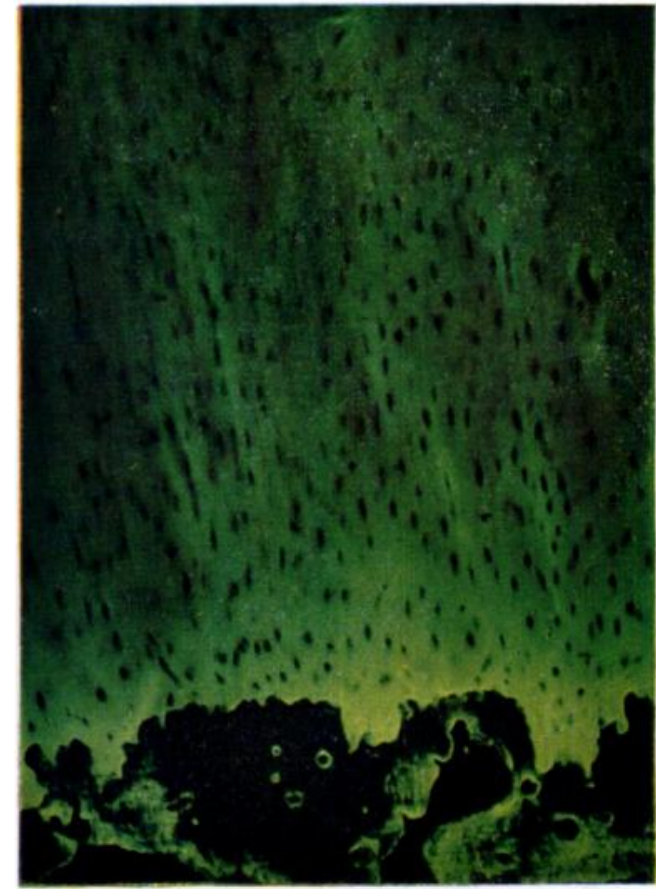

FIG. 4

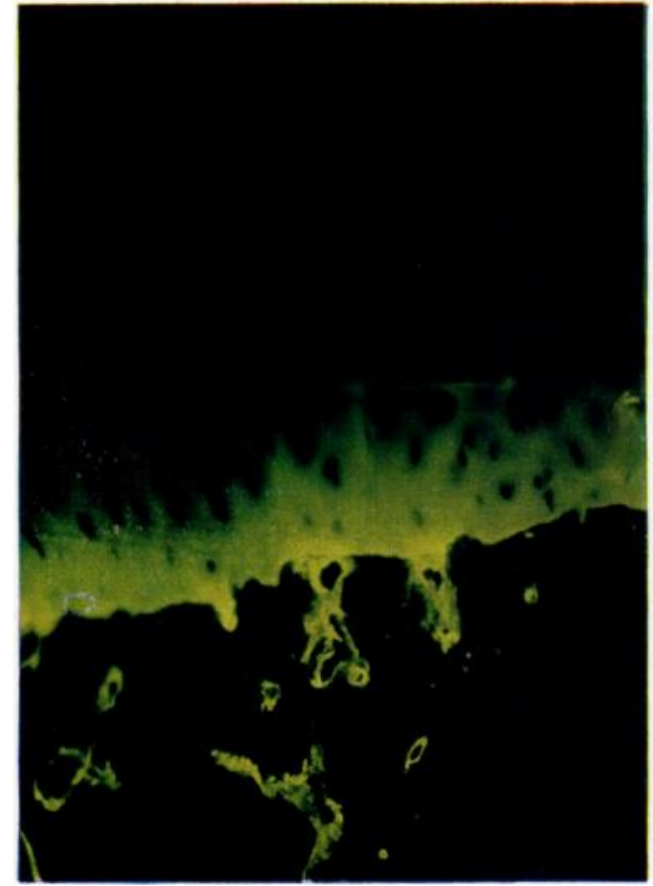

FIG. 5

Figure 4-An undecalcified cryostat section of human articular cartilage with subchondral structure (at the bottom) viewed with ultra-violet light after a forty-eight-hour penetration period. Fluorescence is seen throughout the articular cartilage. ( 30.) Figure 5-An undecalcified cryostat section of human articular cartilage and subchondral bone viewed with ultra-violet light after a sixteen-hour penetration period. Fluorescence is seen only in the articular cartilage and in the collagen surrounding the vascular channels which are present in the calcified cartilage. $(\because 30$.

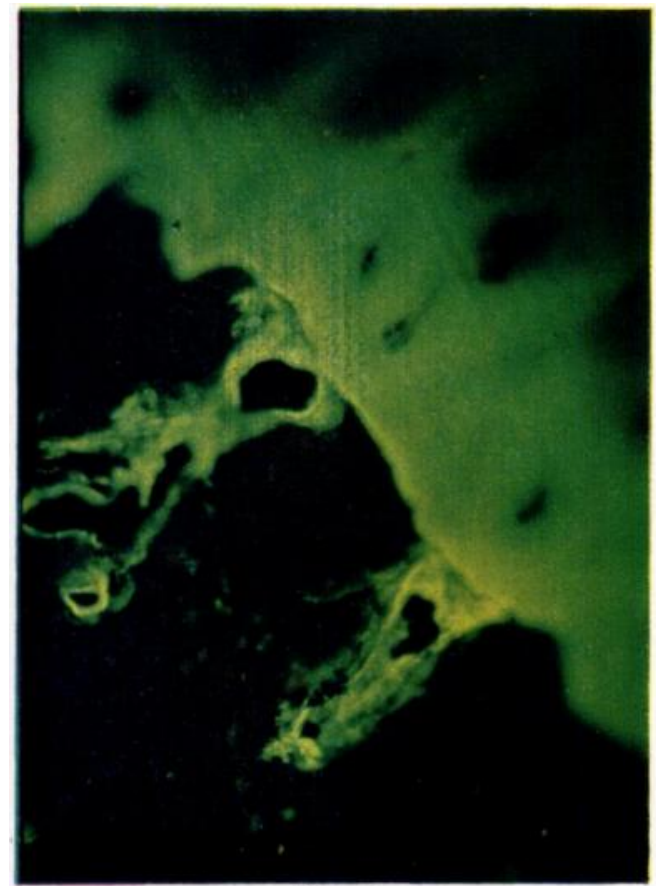

FIG. 6

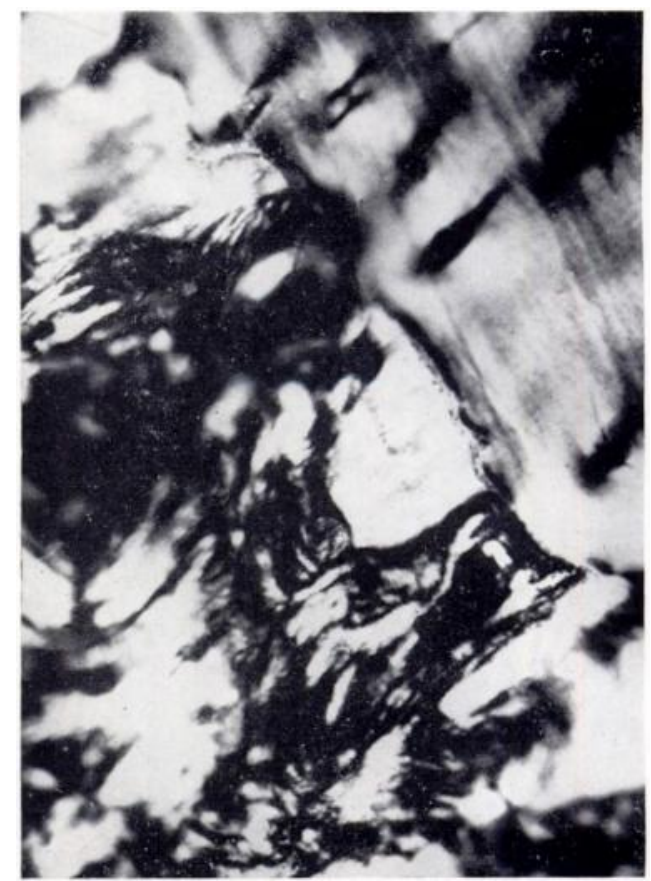

Fig. 7

Figure 6-The same as Figure 5. Fluorescence is seen linking the vascular channels with the articular cartilage. $(\times 120$.$) Figure 7$-The same as Figure 6 but viewed with polarised light. Two vascular channels are seen penetrating the calcified cartilage and making contact with the articular cartilage at the decalcified line. $(\because 120$. 


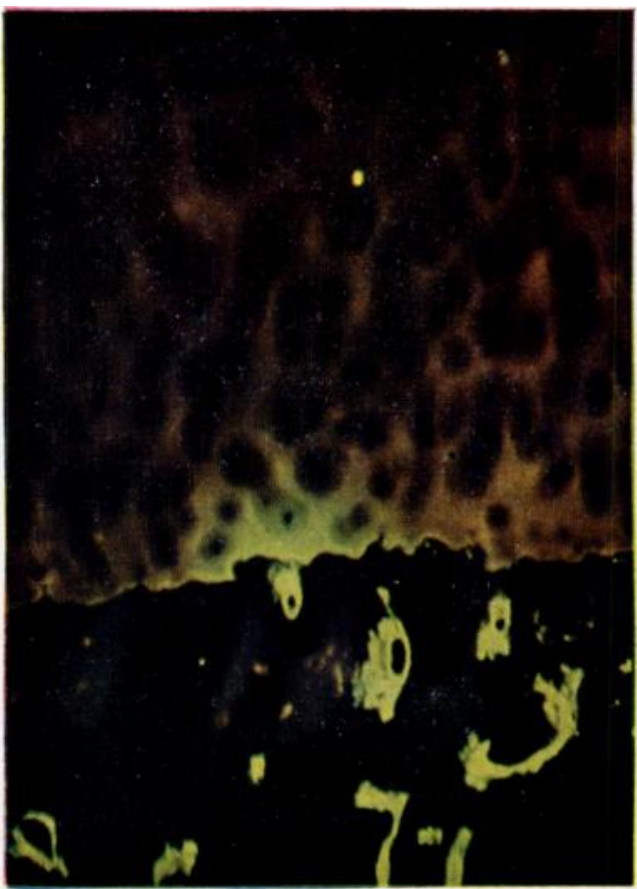

FIG. 8

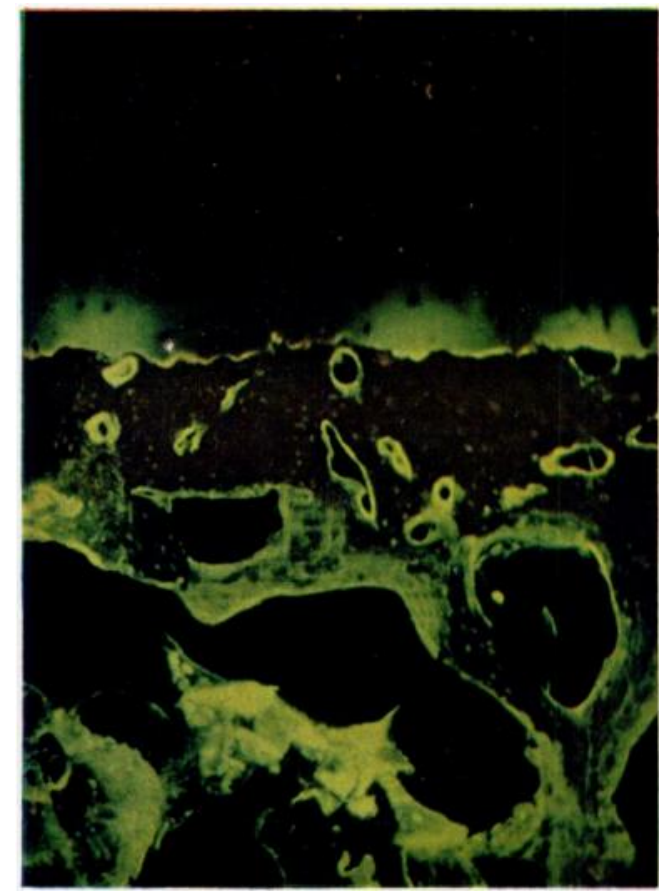

FIG. 9

Figure 8-Undecalcified cryostat section from an osteoarthritic femoral head viewed with ultra-violet light after a forty-eight-hour penetration period. Slight fluorescence is observed in the articular cartilage only in the area where there is an adjacent vascular channel. (30.) Figure 9-Undecalcified cryostat section of a human acetabulum viewed with ultra-violet light after a sixteen-hour penetration period. The subchondral plate is thicker than that of the femoral heads and fluorescence in the articular cartilage is very slight and is only in areas related to vascular channels. $(30$.)

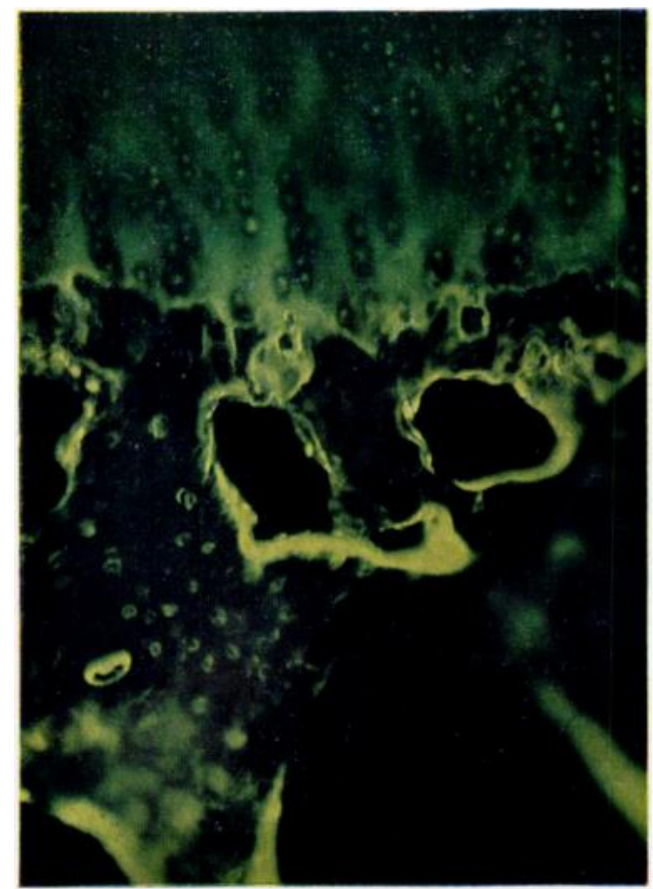

FIG. 10

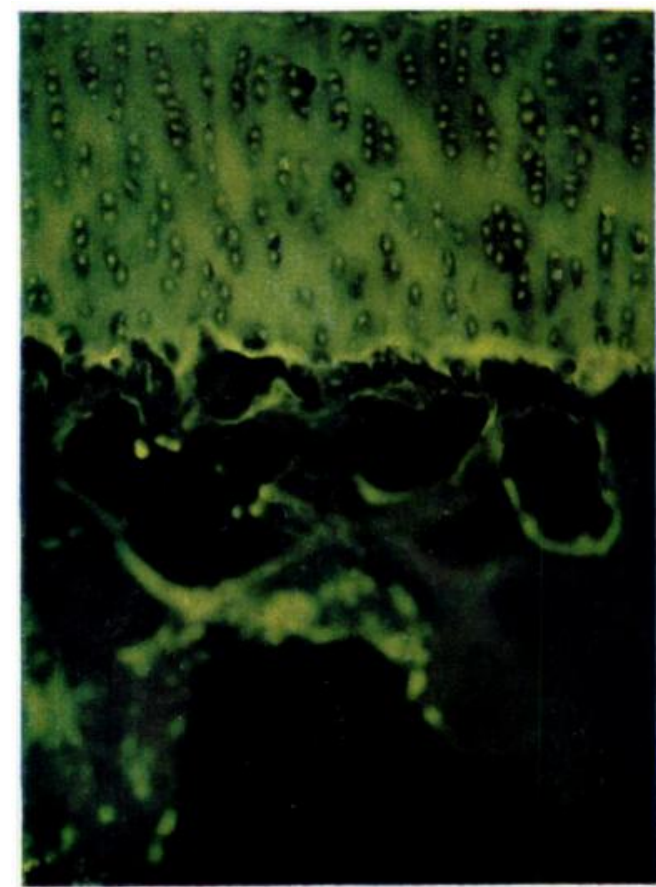

FIG. 11

Undecalcified cryostat sections of eight-month-old rabbit fe:noral head viewed with ultra-violet light after a one-hour penetration period. Fluorescence is observed in the articular cartilage of the dislocated femoral head (Fig. 10) and is present in greater intensity in the control head (Fig. 11). (×120.)

VOL. 51 B, NO. 4, NOVEMBER 1969 
The sulphur ${ }^{35}$ experiments on the femoral heads showed that penetration had occurred through the subchondral plate and into the entire depth of the articular cartilage.

The in vivo experiments showed fluorescence in the articular cartilage of both femoral heads of the rabbit. The intensity of fluorescence in the dislocated side was less than that of the control (Figs. 10 and 11).

\section{DISCUSSION}

The results from the forty-eight-hour experiments confirm that a pathway for dye from the medullary cavity to the articular cartilage in the human femoral head does exist. In the sixteenhour experiments the migration of the dye was confined to areas where there were vascular channels near the articular cartilage. The osteoarthritic femoral head and acetabula had fewer vascular channels and it was easy to detect that the dye had only penetrated into the articular cartilage through these channels. This study clearly suggests that these vascular channels may play a part in the nutrition of the adult human articular cartilage.

The rabbit experiments confirmed that the dye could diffuse into the articular cartilage from the vascular compartment. The rabbits did not have a fully mature femoral head but the dye had passed from the medullary cavity into the articular cartilage in areas where there were vascular spaces.

The comparison of histological preparations of rabbit and human femoral heads shows consistent anatomical differences which are important in the present context. In the immature rabbit, where the capital epiphysis is still growing, vascular channels extend into the articular cartilage (Fig. 12). There is calcification of the articular cartilage where vascular penetration is occurring. When growth of the capital epiphysis stops, as in the mature rabbit, the articular cartilage of the femur is separated from the medullary cavity by calcified cartilage or subchondral plate or both (Fig. 13). There were no vascular channels penetrating into the articular cartilage in any of the mature rabbit femoral heads studied. The potential route for the passage of dye or radioactive sulphur in the mature epiphysis is therefore different in rabbit and human material. Results obtained in the mature rabbit should not be freely related to the human condition.

Macroscopic detection does not allow accurate observation of the relationship between microscopic architecture and dye migration. For this reason the selection of a dye which could be easily detected microscopically was mandatory.

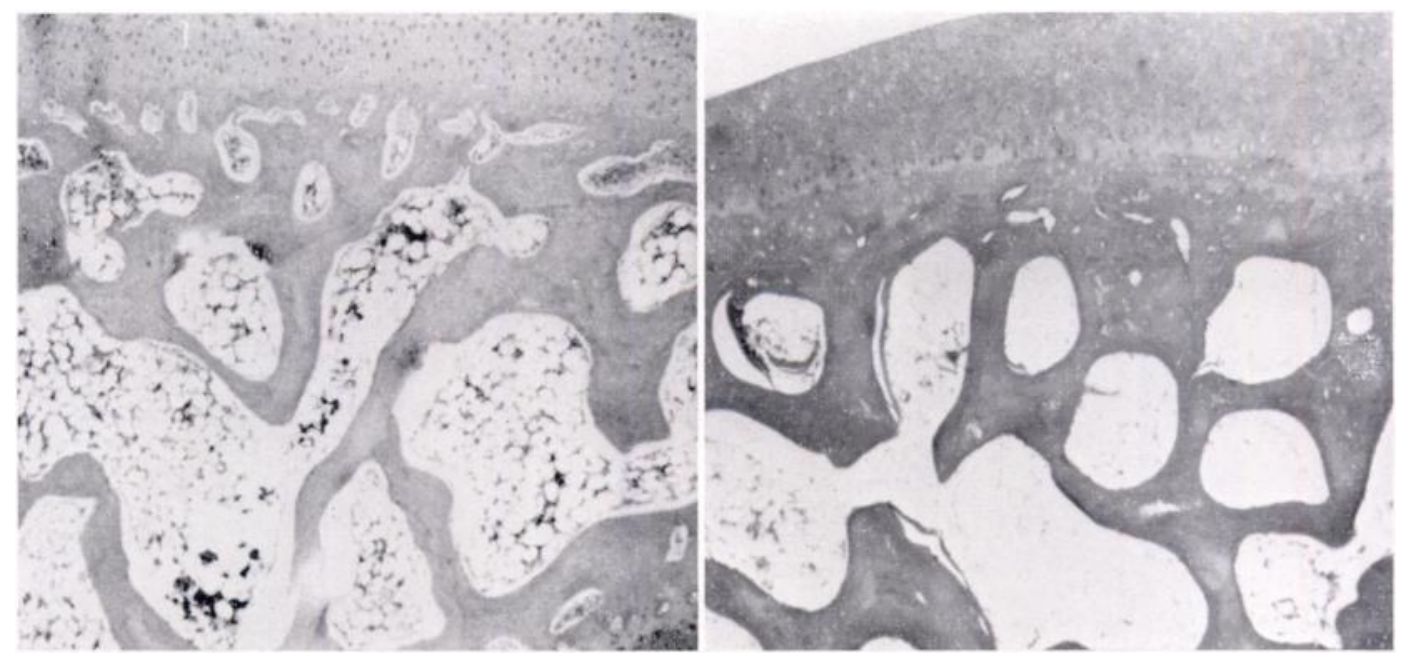

Fig. 12

FIG. 13

Figure 12-Decalcified paraffin section of an eight-month-old rabbit femoral head. A "calcified line" is in evidence and there are vascular spaces extending to it. (Haematoxylin and eosin, $\times 30$.) Figure 13-Decalcified paraffin section of an eighteen-month-old rabbit femoral head. A well defined "calcified line" calcified cartilage and subchondral plate are seen. There are no vascular spaces extending from the medullary cavity to the articular cartilage. (Haematoxylin and eosin, $\times 30$.) 
Neutral red and methylene blue were considered for use as indicators. These dyes were discounted because, when stained preparations were cut, the concentration of the dye was too low to make accurate observations. Fresh sections, stained by immersion in neutral red, showed that the intensity of staining was greater at the surface of the articular cartilage than at its base. It may be that this selective affinity of the cartilage for neutral red could well explain the observations of previous investigators. The fluorescent dye was selected as an indicator because in fresh sections, stained by immersion, the dye bound itself evenly throughout the cartilage. Extremely small amounts of the dye were easily detectable because of its intense fluorescence. The bone and calcified cartilage displayed no affinity for the dye, whereas osteoid and cartilage did. It was concluded that the dye bound itself to collagen provided it was not calcified. The dye was readily soluble in aqueous solutions and therefore, after initial penetration, it was necessary to maintain the tissue in a frozen state to avoid seepage of dye into unstained areas. Freezing the tissues did not produce architectural artefacts detectable by light microscopy. The dye has a molecular weight of 364 , which is greater than that of known amino acids in collagen. It is assumed that if the large dye molecule can pass through so too could the smaller nutritional amino acids.

\section{SUMMARY}

1. The routes by which adult human articular cartilage can receive its nutrition is still a subject of controversy.

2. Microscopic examination of normal adult human femoral heads has revealed vascular channels which penetrate the subchondral plate and calcified cartilage. These channels bring the medullary soft tissue into contact with the articular cartilage.

3. A fluorescent dye migration technique was used to show that the observed vascular channels are pathways for dye from the medullary cavity to the articular cartilage. It is suggested that these pathways could also be routes by which articular cartilage receives part of its nutrition. 4. The nutritional mechanism in the mature rabbit and adult human femoral heads cannot be compared because histological studies revealed differences in their subchondral structures.

We wish to thank the Arthritis and Rheumatism Council for financial support and also Dr C. G. Woods of the Nuffield Orthopaedic Centre for doing the animal experiments and for helpful discussion.

\section{REFERENCES}

Brodin, H. (1955): Paths of Nutrition in Articular Cartilage and Intervertebral Discs. Acta Orthopaedica Scandinavica, 24, 177.

Brower, T. D., Akahoshi, Y., and Orlic, P. (1962): The Diffusion of Dyes Through Articular Cartilage In Vivo. Journal of Bone and Joint Surgery, 44-A, 456.

Екноцм, R. (1951): Articular Cartilage Nutrition. Acta Anatomica, 11, Supplementum 15.

HodGE, J. A., and McKibBin, B. (1969): The Nutrition of Mature and Immature Cartilage in Rabbits. Journal of Bone and Joint Surgery, 51-B, 140.

Holmdahl, D. E., and INGelmark, B. E. (1950): The Contact between the Articular Cartilage and the Mcdullary Cavities of the Bone. Acta Orthopaedica Scandinavica, 20, 156.

Maroudas, A., Bullough, P., Swanson, S. A. V., and Freeman, M. A. R. (1968): The Permeability of Articular Cartilage. Journal of Bone and Joint Surgery, 50-B, 166.

McKibBin, B., and Holdsworth, F. W. (1966): The Nutrition of Immature Joint Cartilage in the Lamb. Journal of Bone and Joint Surgery, 48-B, 793. 